2017-5

\title{
Surface Acoustic Wave Based Pumping in a Microchannel
}

\author{
Tao Wang \\ University of South Florida, Tampa \\ Qi Ni \\ University of South Florida, Tampa \\ Nathan B. Crane \\ Brigham Young University - Provo, nbcrane@byu.edu \\ Rasim Guldiken \\ University of South Florida, Tampa
}

Follow this and additional works at: https://scholarsarchive.byu.edu/facpub

Part of the Other Mechanical Engineering Commons

\section{Original Publication Citation}

T. Wang, Q, Ni, N. B. Crane, and R. Guldiken, "Surface Acoustic Wave Based Pumping in a Microchannel," Microsystem Technologies, doi:10.1007/s00542-016-2880-9, v 23 n5, p 1335-1342, May 2017.

\section{BYU ScholarsArchive Citation}

Wang, Tao; Ni, Qi; Crane, Nathan B.; and Guldiken, Rasim, "Surface Acoustic Wave Based Pumping in a Microchannel" (2017). Faculty Publications. 5348.

https://scholarsarchive.byu.edu/facpub/5348

This Peer-Reviewed Article is brought to you for free and open access by BYU ScholarsArchive. It has been accepted for inclusion in Faculty Publications by an authorized administrator of BYU ScholarsArchive. For more information, please contact ellen_amatangelo@byu.edu. 


\title{
Surface Acoustic Wave Based Pumping in a Microchannel
}

\author{
Tao Wang ${ }^{\mathrm{a}}$, Qi Ni ${ }^{\mathrm{a}}$, Nathan Crane ${ }^{\mathrm{a}}$ and Rasim Guldiken ${ }^{\mathrm{a}, *}$ \\ ${ }^{a}$ Department of Mechanical Engineering, University of South Florida, Tampa, Florida, 33620, \\ USA \\ *Corresponding author. Tel.: +1-813-974-5628; fax: +1-813-974-3539. \\ E-mail address: guldiken@usf.edu
}

\begin{abstract}
Pumping and manipulation of liquids in microfluidic channels is important for many mechanical, chemical and biomedical applications. Surface acoustic wave based devices fabricated on high-efficiency piezoelectric substrates have been recently investigated for mixing and separation application within microfluidic channels. In this paper, we introduce a novel integrated surface acoustic wave based pump for liquid delivery and precise manipulation within a microchannel. The device employs a hydrophobic surface coating (Cytop) in the device design for decreasing the friction force and increasing the bonding. Contrary to previous surface acoustic wave based pump, this device does not need precise layers of water and glass in the design between substrate and channel, simplifying the design significantly, increasing its application space and mass production potential. In this study, we conducted extensive parametric studies to quantify the effects of volume of the liquid pumped, microchannel size, existence of hydrophobic surface coating as well as the input applied power on the pumping velocity and pump performance. Our results indicate that the pumping velocity for a constant liquid volume with the same applied input power can be increased by over $130 \%(2.31 \mathrm{~mm} / \mathrm{min}$ vs $0.99 \mathrm{~mm} / \mathrm{min})$ by employing a hydrophobic surface coating (Cytop) in a thinner microchannel $(250 \mu \mathrm{m}$ vs $500 \mu \mathrm{m}$ ) design. This device can be used in circulation, dosing, metering and drug delivery applications in which small-scale precise liquid control and delivery is important.
\end{abstract}

Keywords: surface acoustic wave; pumping; manipulation, microfluidic 


\section{Introduction}

Pumping, mixing and separation in microfluidic systems are actively investigated due to increased demand for low cost and portable devices for biomedical, chemical and mechanical applications. For mixing[1,2,3] and separation[4-7] purposes, many fairly high-efficiency devices have been thoroughly investigated. Pumps are one of the key components for delivering and controlling flow in microfluidic systems. Typically, liquids are difficult to pump in microchannels due to low Reynolds number which is well below 10. At low Reynolds numbers, friction forces dominate the pumping forces and liquid appear to be more viscous than it is, resulting in large flow resistances and laminar flow profile[8]. Currently, the most widely used pumping types in microfluidic systems are mechanical and electrical pumps[9,10]. However, mechanical pumps need high power and electrical pumps need high voltage for efficient operation. Surface acoustic wave (SAW) based droplet manipulation at free surface has been reported with high velocity up to several centimeters per second[11]. However, these techniques only work efficiently for pumping liquids on free surfaces (such as droplets), not within a microfluidic channel. The advantages of the SAW pumps as compared to alternatives are that it is a non-contact technique capable of precise and highly repeatable flow manipulation. Also, SAW pumps do not require high power or voltage for high-efficiency operation.

Previous studies reported that SAWs can only pump liquids in a closed microchannel for a very short distance from outside the channel. However, microfluidic applications necessitate not just forcing liquid into a microchannel[12,13] but precise fluid manipulation within a microchannel[14]. There has been a recent surface wave driven pump that accomplished continuous liquid pumping inside a polydimethylsiloxane (PDMS) closed channel[15]. However, this device needs water and glass layers in the design between substrate and channel. The existence of precisely controlled water and glass layers limits the application space and mass production potential. In this paper, we introduce a novel integrated surface acoustic wave based active pump for liquid delivery and precise manipulation within a PDMS-based microchannel. PDMS is widely used as biocompatible material with advantages of low reflection effect on SAWs, wide temperature range and high optical transparency[16]. The device employs a hydrophobic surface coating (Cytop) on the microchannel for decreasing the friction force and increasing the bonding. When compared to other closed channel micropumps reported, this 
device can precisely manipulate a liquid inside a microchannel without requiring high power input.

\section{Device Operation, Simulation and Design}

Figure 1 illustrates the design concept and working mechanism of the surface acoustic wave (SAW) based fluid manipulation and pumping device investigated in this study. After surface waves are generated by judiciously designed interdigital transducers (IDTs), the waves propagate towards the microchannel from the IDTs and interact with the liquid located in the channel. The compressional component of the wave is diffracted at the Rayleigh angle into the fluid. When the surface acoustic waves travel through the substrate, the temperature increases as the applied voltage is increased[17]. Hence, the IDTs will transfer the heat to the air and liquid inside the microchannel. As the temperature of the air and water is increased due to wave propagation, an expansion force will impact the liquid forcing it to move in the microchannel. It should be noted that even when the inlet is entirely filled with liquid, there is still an expansion force. Hence, the force that causes fluid manipulation in the channel is the resultant force of the body force and expansion force that moves the liquid with low power consumption.

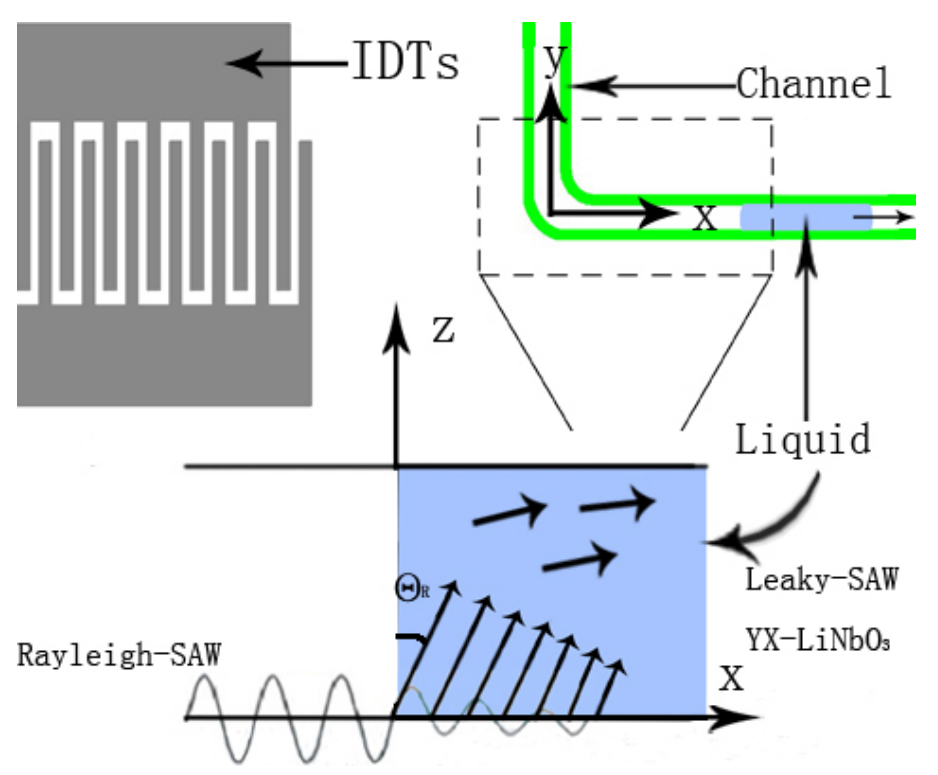

Figure 1. Conceptual view of the liquid pumping and manipulation induced by surface acoustic waves. 
Finite element simulations (FEM) was conducted as a part of this study to optimize the design parameters by using the commercial COMSOL Multiphysics 4.4 software[18,19]. As illustrated in Figure 2, we simulated only a segment of the entire SAW device structure consisting of single wavelength portion to simplify the geometry and reduce the computing power. In the simulations the structure and electric potential boundaries $\left(\Gamma_{\mathrm{L}}\right.$ and $\Gamma_{\mathrm{R}}$ as indicated in Figure $2 \mathrm{a}$ of the IDT fingers was set as periodic in nature to simulate multiple pairs of IDT fingers with a simplified geometry. As expectedly, the SAW velocity will change as a function of the finger thickness and its material. Hence, we investigated the effect of the gold and chromium finger heights ranging from $90 \mathrm{~nm}$ to $1 \mu \mathrm{m}$ on the transducer frequency response. An optimized layer thickness and materials will result in operation frequency closer to the design frequency.

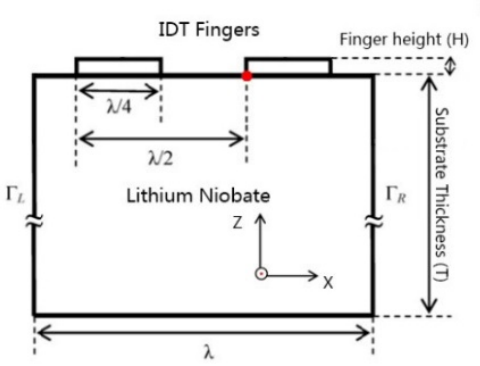

a

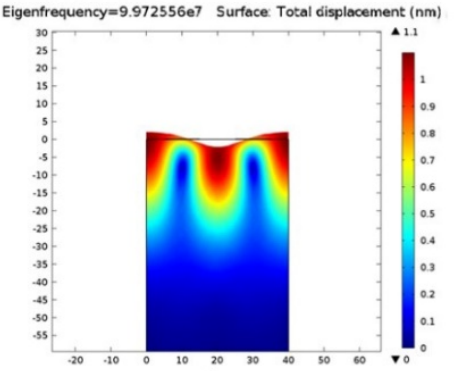

b

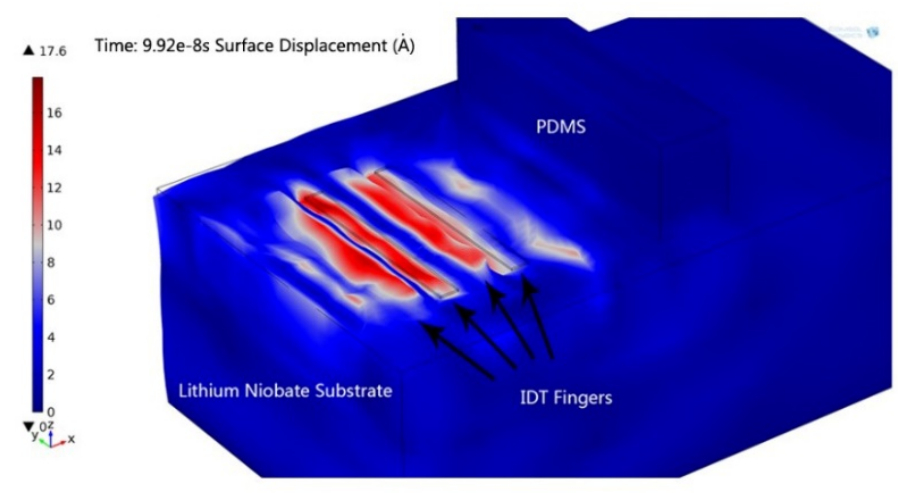

C

Figure 2. (a) SAW transducer geometry employed in the simulations; (b) profile of the IDT fingers formed by $100 \mathrm{~nm}$ chromium deposited on a $500 \mu \mathrm{m} 127.8^{\circ}$ Lithium Niobate substrate (c) 3D Simulation result demonstrating the wave propagation from the IDT transducers towards the PDMS microchannel 
First group of simulations was conducted employing the eigenfrenquency module of the COMSOL to obtain the effect of different heights and materials on the design frequency (Figure $3 a$ ). For a constant substrate thickness of $500 \mu \mathrm{m}$ and wavelength ( $\lambda$ as indicated in Figure 2a) of $40 \mu \mathrm{m}$, the theoretical design resonance frequency turns out to be $99.75 \mathrm{MHz}(\mathrm{f}=\mathrm{Vsaw} / \lambda)$. It should be highlighted that the effects of finger height and substrate thickness are not captured in this theoretical resonance frequency calculations that is employed by many researchers around the world. However, as can be clearly observed from the Figure 3a, the operation frequency of the device is a function of the IDT finger height. In fact, if one employs Gold (Au) as the Chromium (Cr) as the adhesion layer, the operation frequency is a significant function of the finger height. For instance, if one uses a $0.5 \mu \mathrm{m}$ thick finger in the design, the actual operation frequency is shifted to $95.5 \mathrm{MHz}$. It can be observed that employing Chromium as the IDT finger material results in far less variation in the operation frequency for the entire range of finger heights investigated in this study.

A second group of simulations were conducted to investigate the effect of substrate thickness on the operation frequency of the SAW devices (Figure 3b). One can observe from this figure that the effect of substrate thickness on the device operational frequency is minimal. In fact, this is an important result in that many of the wafer substrate manufacturers do not specify the exact wafer thickness but rather substrate thickness range (such as $500 \mu \mathrm{m}-550 \mu \mathrm{m}$ ). Hence even with the existence of this variation, the operational frequency stays almost constant. It should be highlighted that these simulations were conducted for $100 \mathrm{MHz}$ operation frequency, for applications requiring high frequency operation (such as in the $\mathrm{GHz}$ range), the effect of substrate thickness may in fact become more significant. Based on these simulation studies, we employed $100 \mathrm{~nm}$ chromium IDT finger material patterned on a $\sim 500 \mu \mathrm{m}$ Lithium Niobate substrate. 
a)

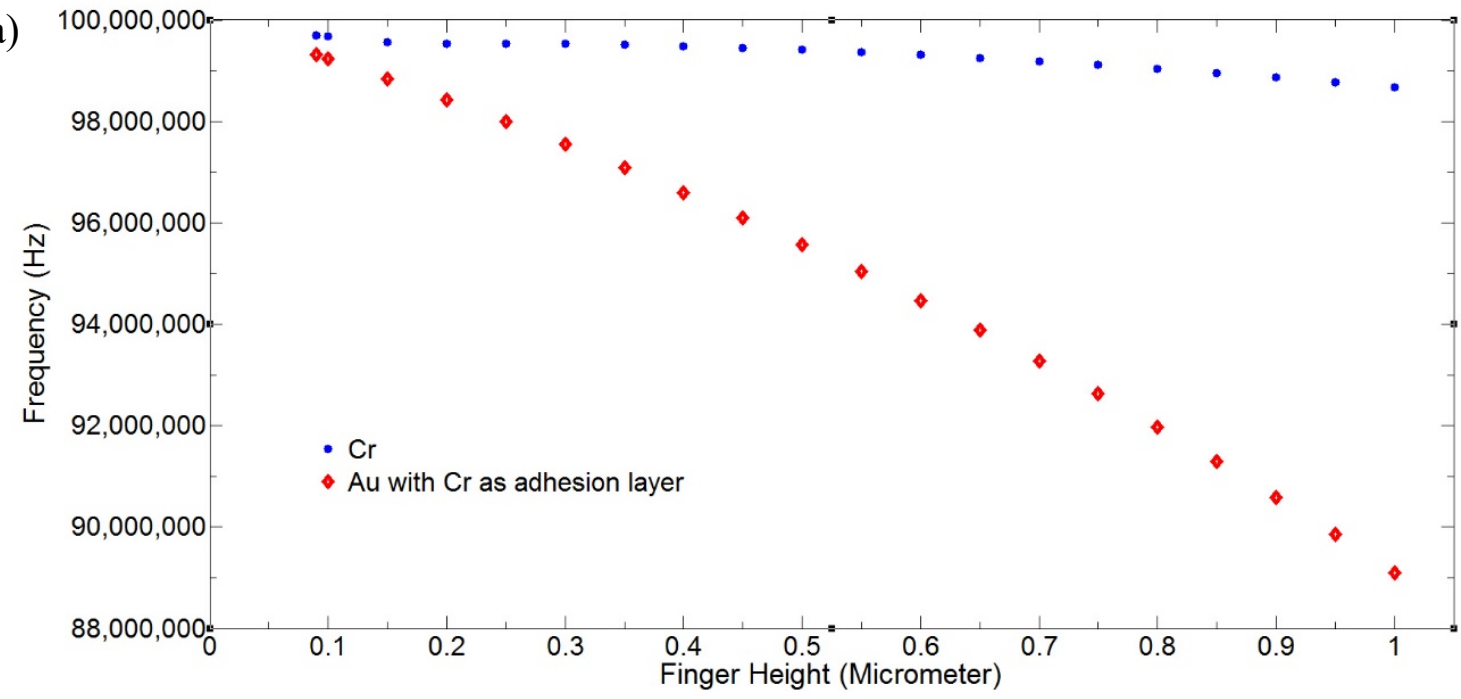

b)

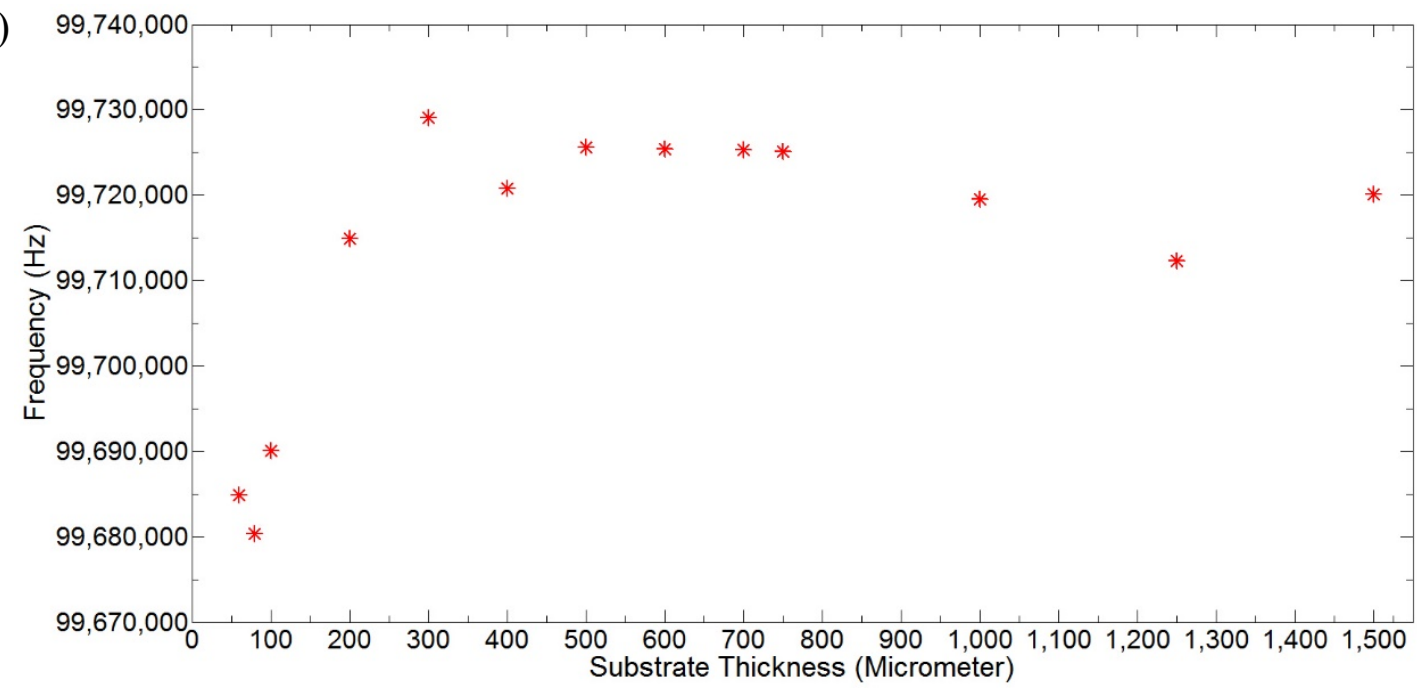

Figure 3. (a) Simulated operation frequency as a function of the IDT finger height and material selection; (b) simulated operation frequency as a function of the substrate thickness

Table 1 illustrates the final design parameters of the IDTs used in this study. The SAW wavelength, the IDT finger pitch, finger width and aperture were designed as $40 \mu \mathrm{m}, 40 \mu \mathrm{m}, 10$ $\mu \mathrm{m}$ and $5 \mathrm{~mm}$, respectively. Two different channel widths were used: $500 \mu \mathrm{m}$ and $250 \mu \mathrm{m}$. The channel height was kept constant at $70 \mu \mathrm{m}$. Different channel widths were investigated to quantify the effect on the liquid velocity. The resonance frequency of the SAW is determined by the ratio of the SAW velocity on the substrate and SAW wavelength $(\lambda) ; f=V$ saw $/ \lambda$. 
Table 1. Device parameters used for the liquid pumping experiments inside the PDMS channel.

\begin{tabular}{|c|c|c|c|}
\hline Wavelength $(\lambda)$ & $40 \mu \mathrm{m}$ & Channel width 1 & $500 \mu \mathrm{m}$ \\
\hline IDT finger width & $10 \mu \mathrm{m}$ & Channel width 2 & $250 \mu \mathrm{m}$ \\
\hline IDT finger pitch & $40 \mu \mathrm{m}$ & Channel height & $70 \mu \mathrm{m}$ \\
\hline IDT finger pairs & 50 pairs & Resonance frequency & $97.75 \mathrm{MHz}$ \\
\hline
\end{tabular}

\section{Transducer Fabrication and Microchannel Integration}

The surface acoustic pump was fabricated in four steps: the fabrication of interdigital transducers (IDTs) on a lithium niobate substrate, the fabrication of the PDMS microchannels, Cytop layer coating, and the bonding of PDMS microchannel to the lithium niobate substrate housing the IDTs. Figure 4 illustrates the fabrication process flow of the SAW pumping device. For forming the IDTs, two-side polished Y-cut X-propagation lithium niobate wafers $\left(128^{\circ}\right.$ $\mathrm{LiNbO}_{3}$, Universitywafer, MA, USA), which has a high electromechanical coupling coefficient increasing the pumping efficiency, were used[20]. After pre-cleaning the Lithium Niobate substrate by rinsing with acetone, methanol and de-ionized water; a $100 \mathrm{~nm}$ thick chrome layer was deposited by using the CRC sputtering system. Then, a $1.6 \mu \mathrm{m}$-thick photoresist layer (S1813, Shipley, Marlborough, MA, USA) was spun on the Lithium Niobate wafer. After exposure and developing, the chrome was etched by using the chrome etchant (CR-7S, Cyantek, Fremont, CA, USA). The chrome IDTs processing step was then completed after the final step of stripping the photoresist. 


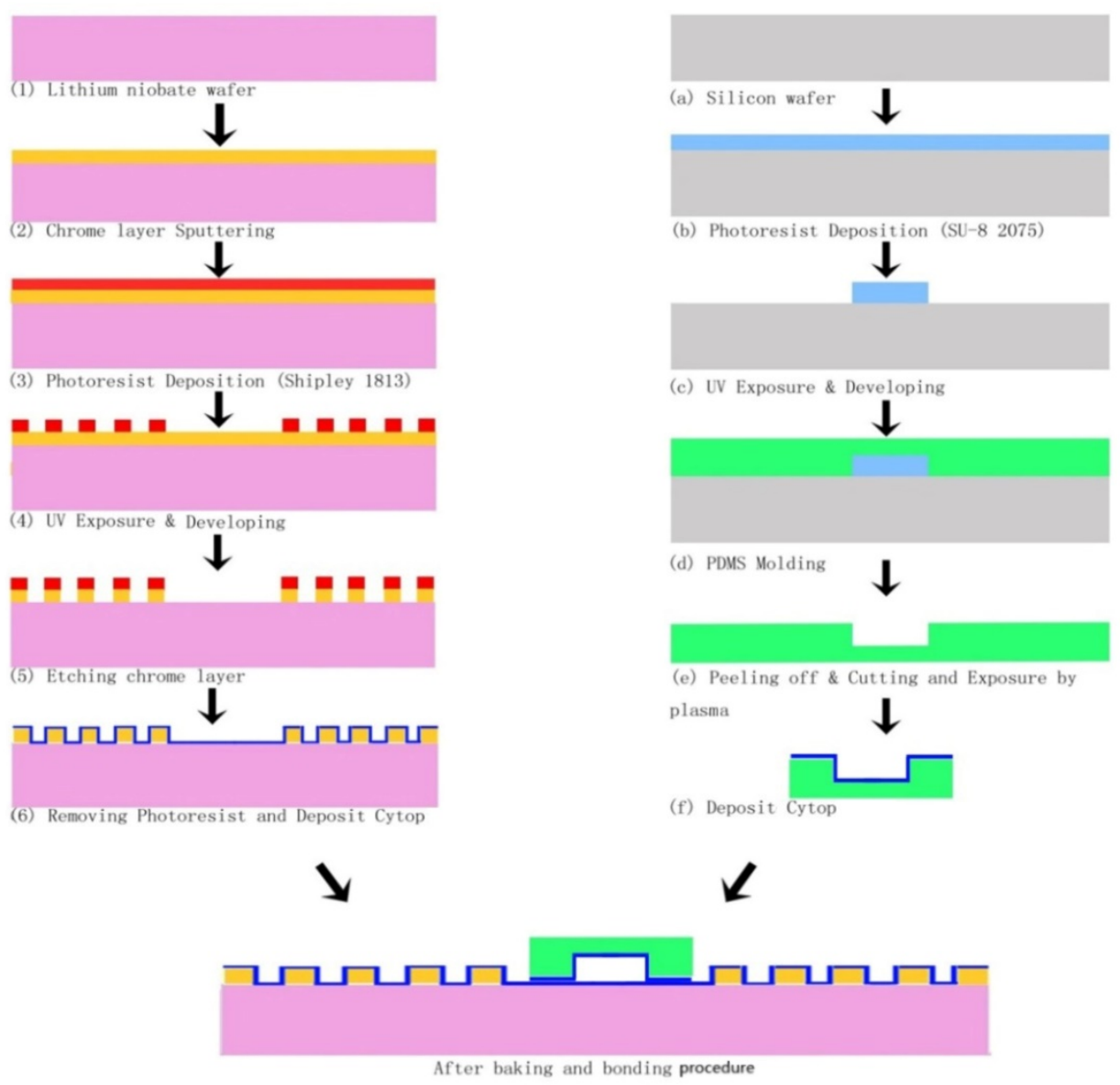

Figure 4. Fabrication process flow for SAW transducers, PDMS molding, Cytop layer spinning and bonding. All steps were completed in the cleanroom facilities of the University of South Florida.

The microchannel was fabricated by PDMS micro molding technique. After the channel mold is formed, PDMS (Sylgard ${ }^{\mathrm{TM}} 184$ kit Dow Corning, Midland, MI, USA) is poured onto the SU-8 mold. It is then cured at room temperature for $24 \mathrm{~h}$ to $48 \mathrm{~h}$ to prevent shrinking of the PDMS. Bonding of the IDT substrate and microchannel as well as the Cytop layer deposition was conducted after separate fabrication of the IDTs and microchannel. The PDMS channel was treated with oxygen plasma first; then both the IDT substrate and the PDMS channel was coated with Cytop ${ }^{\circledR}$ 809M (ASAHI GLASS Co. Ltd, Japan) via spin coating with the average thickness of $1.8 \mu \mathrm{m}$ on the substrate. After separately pre-baking in an oven at $100{ }^{\circ} \mathrm{C}$, a gauge needle was used to create the inlets and outlets. The Cytop layer was annealed at $160^{\circ} \mathrm{C}$ for 1 hour as a final 
step[21]. Finally, the PDMS and the substrate were bonded and uniform pressure was applied. The fabricated IDTs and channel feature on the substrate is illustrated in Figure 5.

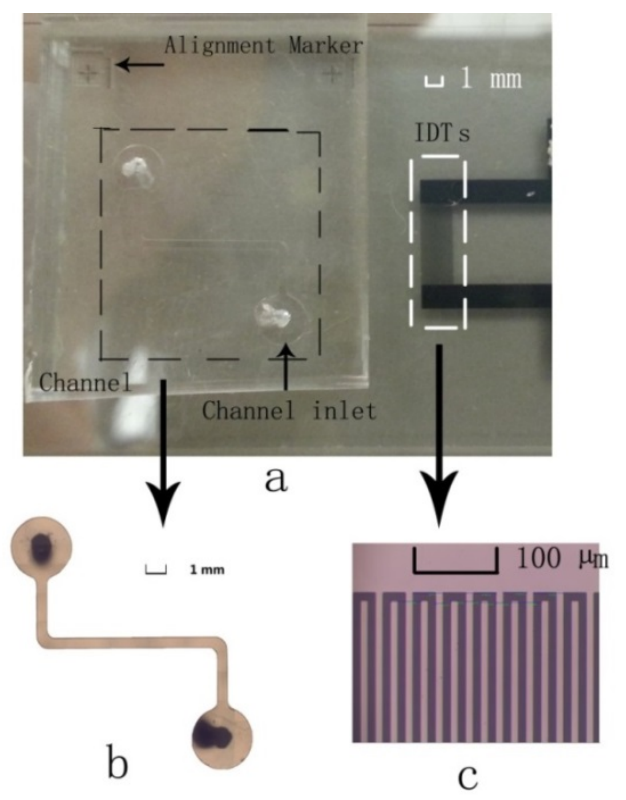

Figure 5. (a) Aligned IDTs and microchannel on the substrate; (b) the closeup of the microchannel design investigated in this study; (c) the details of the chrome IDTs

\section{Experimental Setup}

An inverted microscope (IX-51, Olympus) with a CCD camera was used to visualize and record the experimental data collected (Figure 6). De-ionized water was injected into the channel from the inlet by an external syringe pump (KDS200, KD Scientific, Holliston, MA, USA). RF power amplifier (325LA, ENI) was used to amplify an AC signals generated by a signal generator (AFG3022B, Tektronix). Displacement inside the microchannel was recorded by a CCD camera at $21 \mathrm{fps}$ with a resolution of $1376 \times 1038$. 


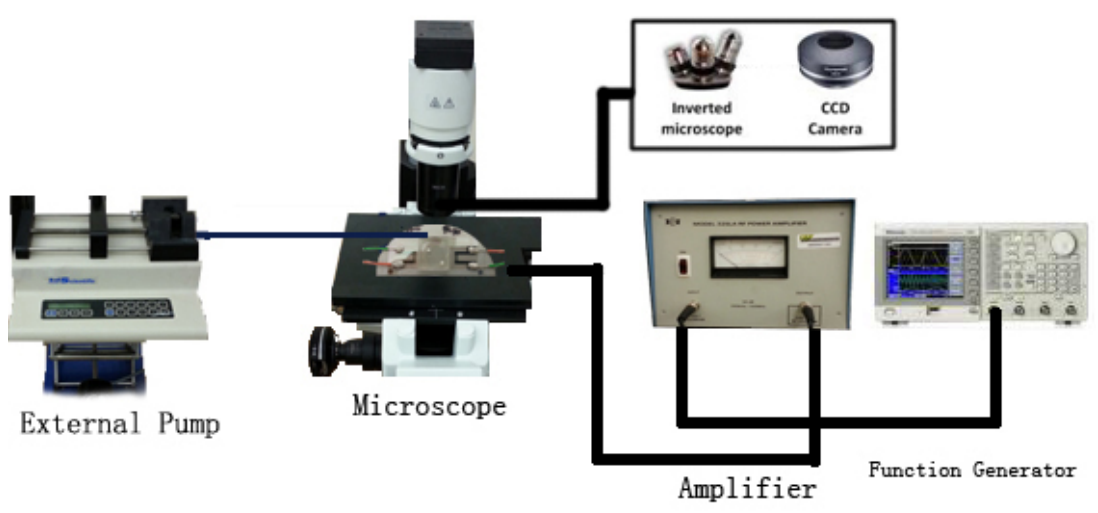

Figure 6. Experimental setup: An external pump was connected to the channel inlet to pump the liquid into the channel. A function generator supplied a continuous sine wave to the amplifier which is connected to the IDTs. Inverted Microscope with CCD camera was used to visualize and record the liquid pumping.

As the total input power is related to the amplitude of the surface acoustic wave generated which determines the pumping velocity[11], a wide-range of applied input power values (ranging from 0 to 7.5 watts) was investigated to obtain the velocity dependence on input power. Also, in order to investigate the effect of liquid volume on the pumping velocity, two different representative volumes $(0.22 \mu \mathrm{l}$ and $0.11 \mu \mathrm{l})$ were used. Additionally, channel widths of $250 \mu \mathrm{m}$ and $500 \mu \mathrm{m}$ were used to investigate the effect of channel width on pumping velocity.

In order to move a droplet through the channel, the pumping system must overcome both the viscous losses in the fluid and the surface tension forces on the substrate. On most surfaces, there is a difference between the contact angle of an advancing liquid/solid interface and a receding interface. The difference in this angle, the contact hysteresis, is related to the energy required to move the contact line by

The contact angle of water on PDMS, which is hydrophobic, is around $110^{\circ}$ for static drops[22]. However, PDMS can have a large contact angle hysteresis such that fluid tends to stick to the PDMS surface. The conventional syringe pump method was used to show the large adhesion force between the fluid and the PDMS surface after plasma bonding. As seen in Figure 7, a large difference between the advancing and receding contact angles was observed as previously reported [23]. The difference between the two angles is commonly known as contact 
angle hysteresis which can cause a strong adhesion force that will prevent the motion of the fluid or droplet. To prevent this, a layer of Cytop coating was introduced to the design. Cytop is a low energy fluorupolymer commonly used to increase the hydrophobicity of surfaces. It is be deposited by spincoating and dipcoating. The Cytop Coating reduces the contact angle hysteresis from $>30^{\circ}$ to $<12^{\circ}$. This difference should substantially reduce the energy required to move the droplet contact line along the channel so that for the same SAW input power, the droplet velocity will be significantly increase.

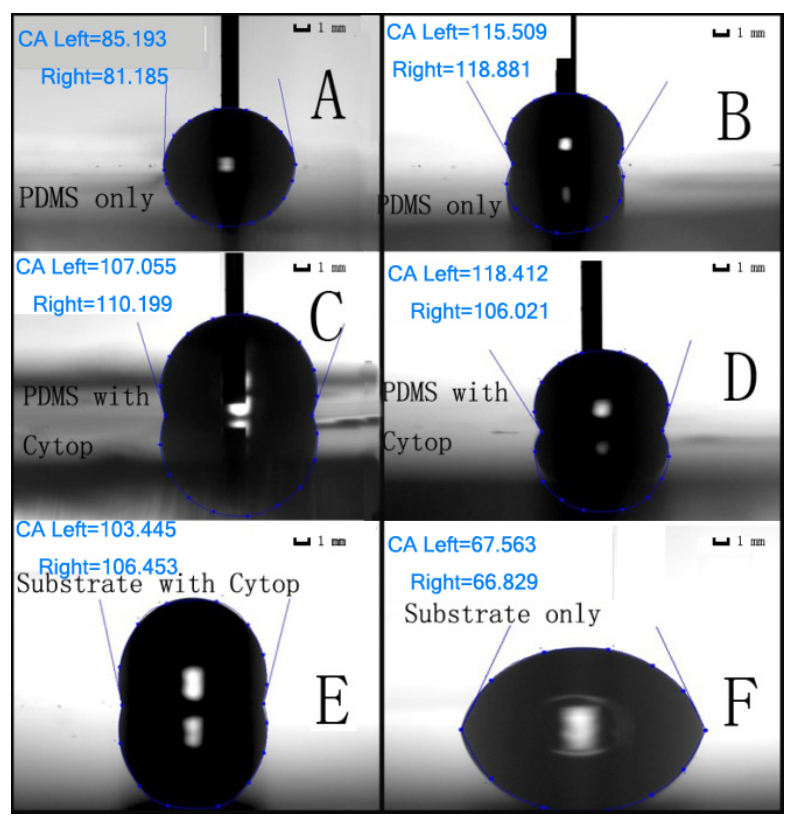

Figure 7. (A) the receding contact angle of the drop on the PDMS surface without Cytop layer; (B) the advancing contact angle of the drop on the PDMS surface without Cytop layer; (C) the receding contact angle of the drop on the PDMS surface with Cytop layer; (D) the advancing contact angle of the drop on the PDMS surface with Cytop layer; (E) the static contact angle of the drop on the substrate with Cytop layer; (F) the static contact angle of the drop on the lithium niobate substrate surface without Cytop layer. The volume of the droplet was kept constant at $10 \mu 1$ in all the experiments.

\section{Results and Discussion}

The liquid pumping was accomplished in this study with IDTs placed 5mm away from the microchannel sidewalls. Initial experiments were conducted with both the inlet 
and outlet open (Figure 8). After the SAWs were excited with a continuous sine wave, the liquid initially started to eject droplets at the low power condition (commonly referred as atomization) as illustrated in Figure 9. As the input power was increased, expectedly, the ejected droplet diameter started increasing[24]. At around 3 watts of input power, in addition to atomization, the liquid started to move and at 6 watts of input power, the atomization stopped while the liquid was being pumped. Figure 9 illustrates the pumping experiment in the $250 \mu \mathrm{m}$ channel without Cytop layer in the device design over 75 seconds. The power applied for this case was 6.5 watts and liquid volume was $0.11 \mu 1$.

a) Sealed inlet $\rightarrow$

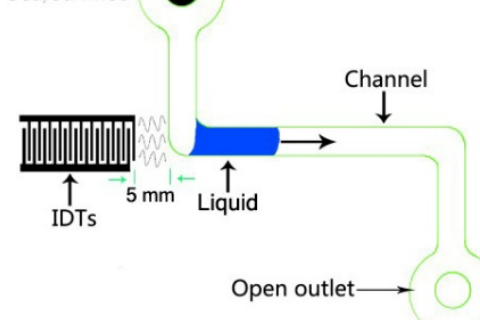

b)
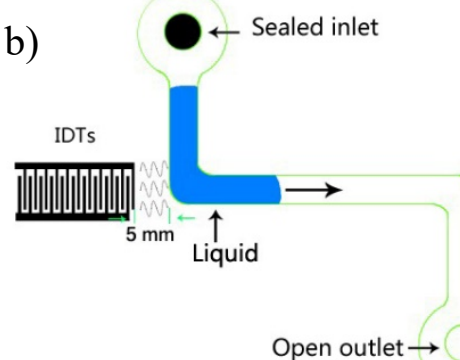

Figure 8. An illustration of different volume of liquid pumping in the channel with sealed inlet and open outlet. Liquid volume in Figure $8 \mathrm{a}$ is $0.11 \mu 1$, while it is $0.22 \mu 1$ in Figure $8 b$.

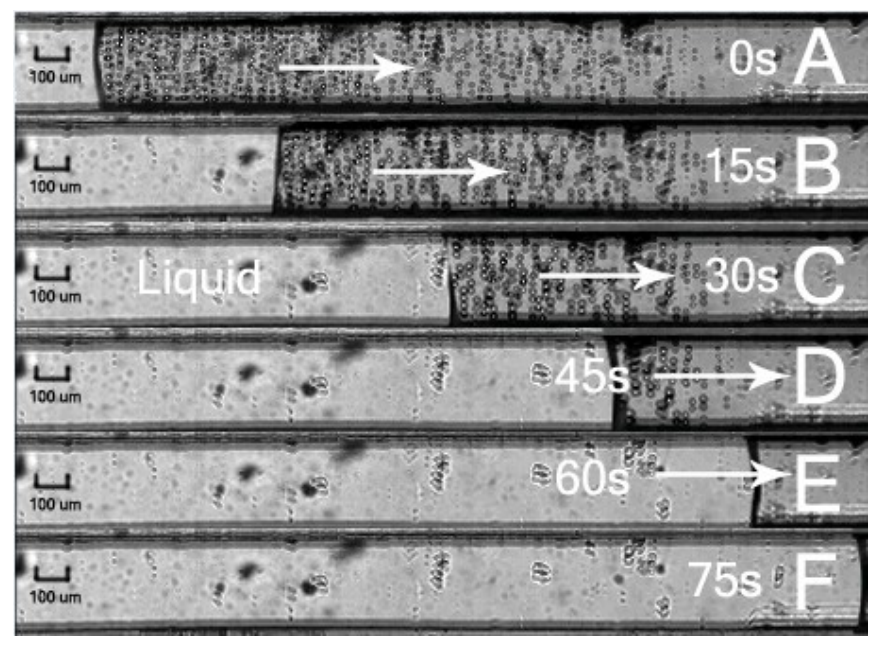

Figure 9. The pumping experiment in the $250 \mu \mathrm{m}$ channel without Cytop over 75 seconds. The power is 6.5 watts and liquid volume is $0.11 \mu 1$. Due to the limitation of the field of view of the microscope, only partial channel is shown. 
Additional sets of experiments were conducted by sealing the inlet of the microchannel with an off-the-shelf masking tape as illustrated in Figure 8. The volume of the sample liquid was $0.11 \mu \mathrm{l}$ and $0.22 \mu \mathrm{l}$ in Figure $8 \mathrm{a}$ and $8 \mathrm{~b}$, respectively. The applied input power ranged from 2 to 6.5 watts and two types of surface coating were used (Lithium Niobate substrate without any coating and Lithium Niobate substrate and channel walls coated with Cytop). Sealing the inlet resulted in heat accumulation over the inlet area and part of the channel which lead the liquid and constrained air to expand. As a result, the driving force was composed of acoustic body force as well as an expansion force. Increasing the input power resulted in increasing the heat generation as well as the acoustic wave amplitude which lead to increasing the acoustic body force. Hence, the applied input power was found to be proportional to the pumping velocity. Figure 10 illustrates the pumping velocity as a function of applied input power with two different liquid volumes in a $500 \mu \mathrm{m}$ microchannel with the Cytop layer in the design. Expectedly, increasing the input applied power resulted in increasing the pumping velocity for both liquid volume cases investigated. One can observe from individual experimental data points that for applied input power range from 2 to 3 watts, the pumping velocities were not a strong function of the volume of the liquid being pumped. Note that this power range corresponds to pumping of the liquid sample with atomization present. However, the larger liquid volume case $(0.22 \mu 1)$ resulted in larger pumping velocity as compared to the lower liquid volume case $(0.11 \mu \mathrm{l})$ once the power is increased further. Higher pumping velocity is obtained with higher liquid volume case for the same power input because the liquid in the higher volume case is exposed to larger body force in the longitudinal direction (along the length of the microchannel). Also, as can be observed from Figure 8, larger volume case has smaller inlet space available for expansion pushing the liquid in the desired direction in the microchannel. 


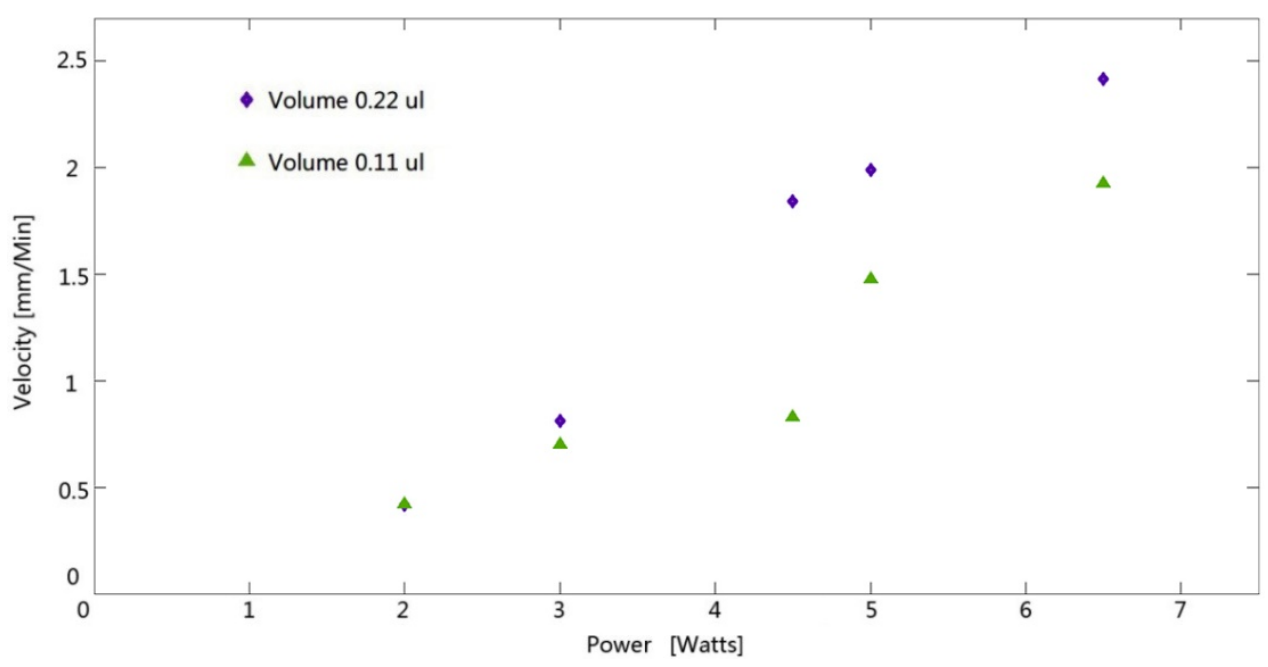

Figure 10. Experimental pumping velocity as a function of applied input power with two different liquid volumes $(0.22 \mu \mathrm{l}$ and $0.11 \mu \mathrm{l})$ in a $500 \mu \mathrm{m}$ microchannel with the Cytop layer coating in the design.

Also, extensive experiments were conducted to investigate the effect of the coating of the microchannel surfaces on pumping velocity. Figure 11 illustrates the experimentally obtained pumping velocity as a function of applied input power for a $500 \mu \mathrm{m}$ microchannel with/without the Cytop layer in the design for two different liquid volumes: $0.22 \mu 1$ (Figure 11a) and $0.11 \mu 1$ (Figure 11b). Additionally, Figure 12 shows the experimentally obtained pumping velocity as a function of applied input power for a thinner $(250 \mu \mathrm{m})$ microchannel with/without the Cytop layer in the design for two different liquid volumes: $0.11 \mu 1$ (Figure 12a) and 0.054 $\mu$ l (Figure 12b). As expectedly, increasing the input applied power resulted in increasing the pumping velocity for all microchannel width and liquid volume cases investigated. One can observe from both Figures 11 and 12 that for the same liquid volume and input power applied cases, hydrophobic surface coating (Cytop) increases the pumping velocity for both microchannel widths and liquid volumes investigated. When one compares Figure $11 \mathrm{~b}$ to Figure $12 \mathrm{a}$, the effect of hydrophobic surface coating on the pumping velocity for the same liquid volume for different microchannel widths can be observed. The inclusion of hydrophobic coating in the channel design has an increased effect on the pumping velocity for wider microchannel $(500 \mu \mathrm{m})$ as compared to thinner channel width $(250 \mu \mathrm{m})$. The reason for obtaining higher pumping velocity for thinner microchannel as opposed to wider channel with the same liquid volume is that the 
length of the fluid within the microchannel is longer for the thinner microchannel resulting in increased body force in the longitudinal direction (along the length of the microchannel) causing higher pumping velocity. It should be noted that the effect of the heat expansion force is negligible when channel width is investigated for same liquid volume as the inlet area where the heat expansion force is generated is the same for both channel widths.

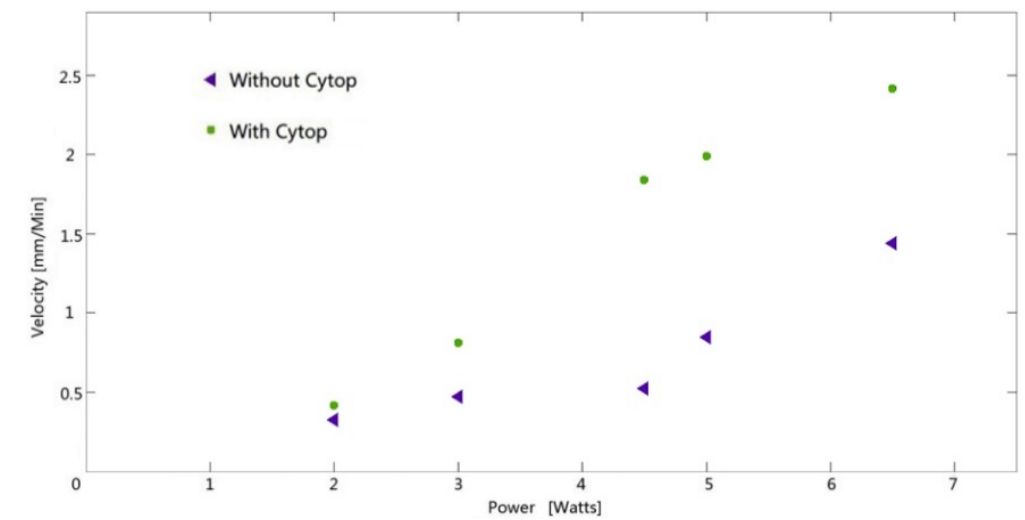

b)

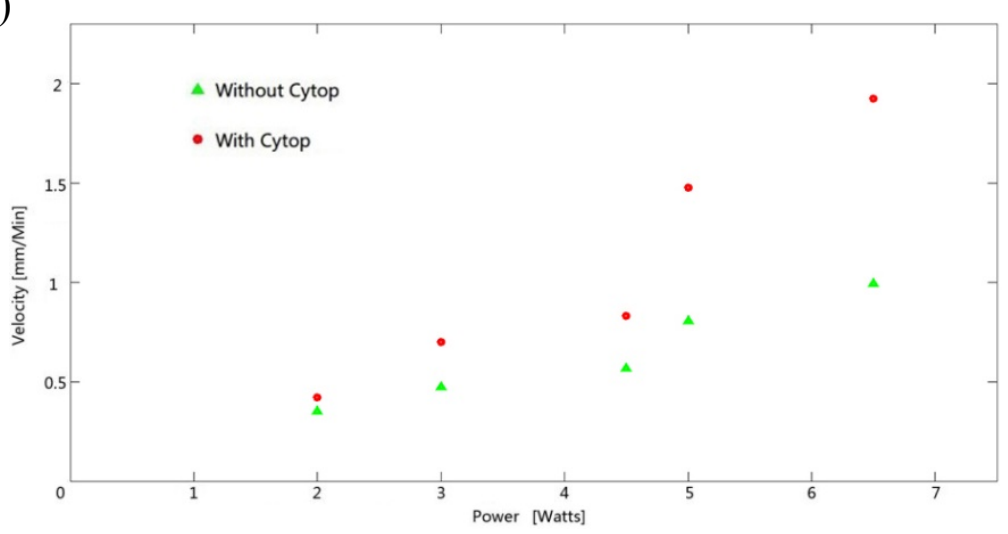

Figure 11. Experimental pumping velocity as a function of applied input power in a $500 \mu \mathrm{m}$ microchannel with/without the Cytop layer in the design for two different liquid volumes: $0.22 \mu 1$ (Figure 11a) and $0.11 \mu 1$ (Figure 11b)

a) 

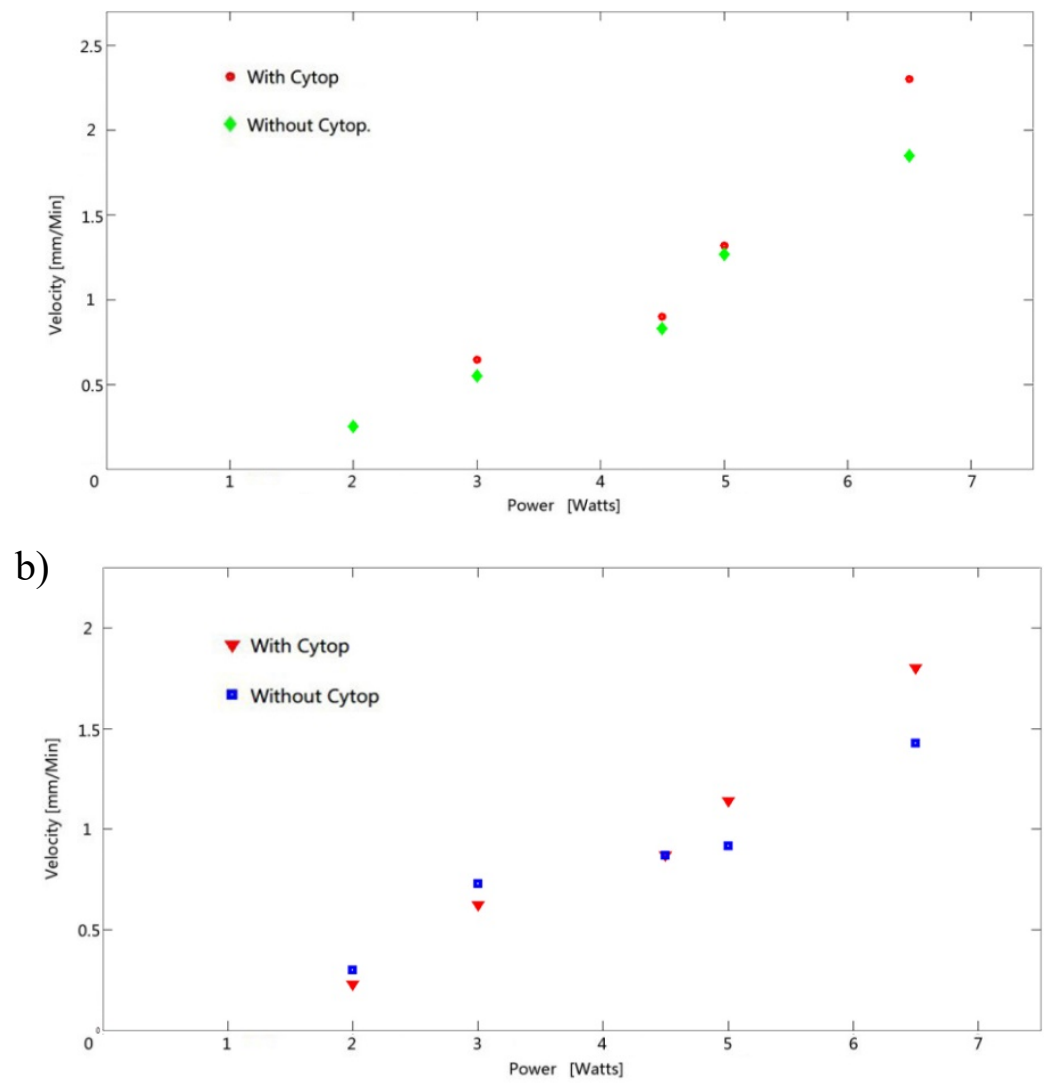

Figure 12. Experimental pumping velocity as a function of applied input power in a $250 \mu \mathrm{m}$ microchannel with/without the Cytop layer in the design for two different liquid volumes: $0.11 \mu 1$ (Figure 12a) and $0.054 \mu 1$ (Figure 12b)

\section{Conclusions}

In this study, we conducted a thorough investigation of liquid pumping within a microfluidic channel by using the phenomenon of surface acoustic wave force and heat expansion force. We illustrated successful pumping within a microchannel with surface acoustic wave devices located outside the microchannel patterned on a piezoelectric substrate. Significant advantages of this device are protection of the liquid within the channel, easy and low cost fabrication, no contact between the pumping device and the liquid, homogenous steady laminar flow and small-scale precise liquid control and delivery. Due to the large friction force of the four microchannel walls surrounding the liquid, a hydrophobic surface coating (Cytop) was investigated in detail and increased pumping velocity with Cytop coating was demonstrated. Our studies indicate that the pumping velocity for a constant liquid volume with the same applied input power can be 
increased by over $130 \%(2.31 \mathrm{~mm} / \mathrm{min}$ vs $0.99 \mathrm{~mm} / \mathrm{min})$ by employing a hydrophobic surface coating (Cytop) in a thinner microchannel $(250 \mu \mathrm{m}$ vs $500 \mu \mathrm{m})$ design. This microchannel pump can be used as circulation, dosing, metering and drug delivery applications in which small-scale precise liquid control and delivery is important. 


\section{References}

[1] M. C. Jo and R. Guldiken, "Dual surface acoustic wave-based active mixing in a microfluidic channel," Sensors Actuators A Phys., vol. 196, pp. 1-7, Jul. 2013.

[2] M. K. Tan, L. Y. Yeo, and J. R. Friend, "Rapid fluid flow and mixing induced in microchannels using surface acoustic waves," EPL (Europhysics Lett., vol. 87, no. 4, p. 47003, Aug. 2009.

[3] K. Sritharan, C. J. Strobl, M. F. Schneider, A. Wixforth, and Z. Guttenberg, "Acoustic mixing at low Reynold's numbers," Appl. Phys. Lett., vol. 88, pp. 1-3, 2006.

[4] T. Dung Luong and N. Trung Nguyen, "Surface Acoustic Wave Driven Microfluidics - A Review," Micro Nanosyst., vol. 2, no. 3, pp. 217-225, Sep. 2010.

[5] R. Guldiken, M. C. Jo, N. D. Gallant, U. Demirci, and J. Zhe, "Sheathless size-based acoustic particle separation.," Sensors (Basel)., vol. 12, no. 1, pp. 905-22, Jan. 2012.

[6] M. C. Jo and R. Guldiken, "Active density-based separation using standing surface acoustic waves," Sensors Actuators A Phys., vol. 187, pp. 22-28, Nov. 2012.

[7] M. C. Jo and R. Guldiken, "Particle manipulation by phase-shifting of surface acoustic waves," Sensors Actuators A Phys., vol. 207, pp. 39-42, Mar. 2014.

[8] J. Teng, "Fluid Dynamics in Microchannels."

[9] R. Shabani and H. J. Cho, "Active surface tension driven micropump using droplet/meniscus pressure gradient," Sensors Actuators B Chem., vol. 180, pp. 114-121, Apr. 2013.

[10] D. J. Laser and J. G. Santiago, "A review of micropumps," J. Micromechanics Microengineering, vol. 14, no. 6, pp. R35-R64, Jun. 2004.

[11] M. Alghane, Y. Q. Fu, B. X. Chen, Y. Li, M. P. Y. Desmulliez, and a. J. Walton, "Streaming phenomena in microdroplets induced by Rayleigh surface acoustic wave," J. Appl. Phys., vol. 109, no. 11, p. 114901, 2011.

[12] M. Cecchini, S. Girardo, D. Pisignano, R. Cingolani, and F. Beltram, "Acousticcounterflow microfluidics by surface acoustic waves," Appl. Phys. Lett., vol. 92, no. 10, p. 104103, 2008.

[13] L. Masini, M. Cecchini, S. Girardo, R. Cingolani, D. Pisignano, and F. Beltram, "Surfaceacoustic-wave counterflow micropumps for on-chip liquid motion control in twodimensional microchannel arrays.," Lab Chip, vol. 10, no. 15, pp. 1997-2000, Aug. 2010. 
[14] S. Girardo, M. Cecchini, F. Beltram, R. Cingolani, and D. Pisignano, "Polydimethylsiloxane-LiNbO3 surface acoustic wave micropump devices for fluid control into microchannels.," Lab Chip, vol. 8, no. 9, pp. 1557-63, Sep. 2008.

[15] L. Schmid, A. Wixforth, D. a. Weitz, and T. Franke, "Novel surface acoustic wave (SAW)-driven closed PDMS flow chamber," Microfluid. Nanofluidics, vol. 12, no. 1-4, pp. 229-235, Aug. 2011.

[16] M. C. Jo and R. Guldiken, "Effects of polydimethylsiloxane (PDMS) microchannels on surface acoustic wave-based microfluidic devices," Microelectron. Eng., vol. 113, pp. 98104, Jan. 2014.

[17] W.-K. Tseng, J.-L. Lin, W.-C. Sung, S.-H. Chen, and G.-B. Lee, "Active micro-mixers using surface acoustic waves on Y-cut $128^{\circ}$ LiNbO 3," J. Micromechanics Microengineering, vol. 16, no. 3, pp. 539-548, Mar. 2006.

[18] A. A. Mohanan, M. S. Islam, S. H. Ali, R. Parthiban, and N. Ramakrishnan, "Investigation into mass loading sensitivity of sezawa wave mode-based surface acoustic wave sensors.," Sensors (Basel)., vol. 13, no. 2, pp. 2164-75, Jan. 2013.

[19] U. C. Kaletta and C. Wenger, "FEM simulation of Rayleigh waves for CMOS compatible SAW devices based on $\mathrm{AlN} / \mathrm{SiO}_{2} / \mathrm{Si}(100)$.," Ultrasonics, vol. 54, no. 1, pp. 291-5, Jan. 2014.

[20] R. Guldiken, M. C. Jo, N. D. Gallant, U. Demirci, and J. Zhe, "Sheathless size-based acoustic particle separation.," Sensors (Basel)., vol. 12, no. 1, pp. 905-22, Jan. 2012.

[21] D. Uchida, S. Sugiura, Y. S. J. S. Go, H. Nakanishi, T. Funatsu, and S. Shoji, "PDMS MICROFLUIDIC DEVICES WITH PTFE PASSIVATED CHANNELS," pp. 3-6, 2003.

[22] A. Mata, A. J. Fleischman, and S. Roy, "Characterization of polydimethylsiloxane (PDMS) properties for biomedical micro/nanosystems.," Biomed. Microdevices, vol. 7, no. 4, pp. 281-93, Dec. 2005.

[23] Q. Dou, C. Wang, C. Cheng, W. Han, P. C. Thüne, and W. Ming, "PDMS-Modified Polyurethane Films with Low Water Contact Angle Hysteresis," Macromol. Chem. Phys., vol. 207, no. 23, pp. 2170-2179, Dec. 2006.

[24] N. Murochi, M. Sugimoto, Y. Matsui, and J. Kondoh, "Deposition of Thin Film Using a Surface Acoustic Wave Device," Jpn. J. Appl. Phys., vol. 46, no. 7B, pp. 4754-4759, Jul. 2007. 